\title{
Local Ablative Strategies for Ductal Pancreatic Cancer (Radiofrequency Ablation, Irreversible Electroporation): A Review
}

\author{
Salvatore Paiella, ${ }^{1}$ Roberto Salvia, ${ }^{1}$ Marco Ramera, ${ }^{1}$ Roberto Girelli, ${ }^{2}$ Isabella Frigerio, ${ }^{2}$ \\ Alessandro Giardino, ${ }^{2}$ Valentina Allegrini, ${ }^{1}$ and Claudio Bassi ${ }^{1}$ \\ ${ }^{1}$ Unit of General Surgery B, The Pancreas Institute, G.B. Rossi Hospital, University of Verona Hospital Trust, Verona, Italy \\ ${ }^{2}$ Pancreatic Surgical Unit, Casa di Cura Pederzoli, Peschiera del Garda, Verona, Italy
}

Correspondence should be addressed to Salvatore Paiella; salvatore.paiella@univr.it

Received 15 July 2015; Revised 28 October 2015; Accepted 13 January 2016

Academic Editor: Bence Sipos

Copyright (c) 2016 Salvatore Paiella et al. This is an open access article distributed under the Creative Commons Attribution License, which permits unrestricted use, distribution, and reproduction in any medium, provided the original work is properly cited.

Pancreatic ductal adenocarcinoma (PDAC) has still a dismal prognosis. Locally advanced pancreatic cancer (LAPC) accounts for the $40 \%$ of the new diagnoses. Current treatment options are based on chemo- and radiotherapy regimens. Local ablative techniques seem to be the future therapeutic option for stage-III patients with PDAC. Radiofrequency Ablation (RFA) and Irreversible Electroporation (IRE) are actually the most emerging local ablative techniques used on LAPC. Initial clinical studies on the use of these techniques have already demonstrated encouraging results in terms of safety and feasibility. Unfortunately, few studies on their efficacy are currently available. Even though some reports on the overall survival are encouraging, randomized studies are still required to corroborate these findings. This study provides an up-to-date overview and a thematic summary of the current available evidence on the application of RFA and IRE on PDAC, together with a comparison of the two procedures.

\section{Introduction}

PDAC is one of the deadliest cancer types. It accounts for about $7 \%$ of all cancer deaths and is actually the fourth cause of cancer death in the United States [1]. Only $20 \%$ of PDAC are resectable at time of diagnosis (with a 5 -year survival of less than $20 \%$ ), while the majority of patients are candidates only for chemotherapy or chemoradiotherapy according to various protocols [2-4]. $40 \%$ of patients are diagnosed with a locally advanced disease, with few chances to undergo surgery even after neoadjuvant treatments. Median overall survival (OS) reported for patients treated with upfront surgery and adjuvant therapy is about 20-22 months [57], while it is about 9.2-11.7 months for stage-III locally advanced pancreatic cancer (LAPC) treated with Gemcitabine alone [8-10] and 9-13 months for patients treated with chemo(radio)therapy [11]. Given that LAPC is nearly the most frequent diagnosis to face and that downstaging occurs only in $10-20 \%$ of patients [12], the novel local therapies, such as Radiofrequency Ablation (RFA) and Irreversible
Electroporation (IRE), have been proposed as new treatment options in the multimodal treatment of the disease [13]. The aim of this paper is to evaluate and compare technical aspects, indications, and results of the application of both RFA and IRE on LAPC.

\section{Physical Bases and Principles of Techniques}

Local thermal or nonthermal techniques are applied to ultimately induce irreversible cellular damage leading to cell death via either apoptosis or coagulative necrosis. Physical bases and principles of technique of both RFA and IRE are briefly shown below.

2.1. RFA. RFA is an ablative therapy that, through the application of a high-frequency alternating current, conveyed by one or more needle electrodes, generates local high temperatures, leading to coagulative necrosis and protein denaturation inside neoplastic tissue. While at temperatures between 60 


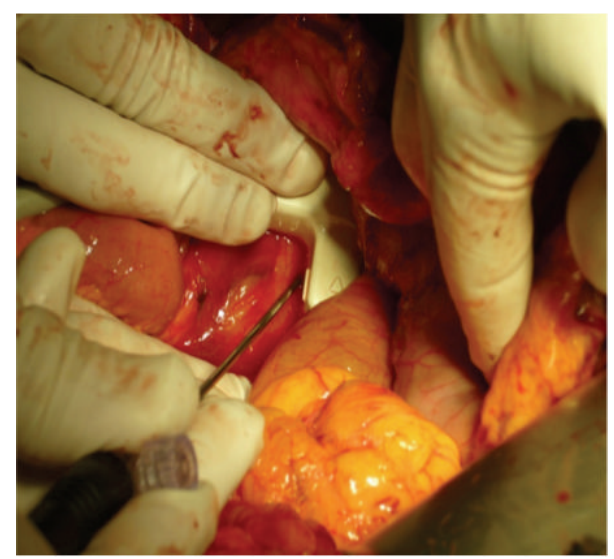

FIGURE 1: US-guided intraoperative application of RFA tip.

and $100^{\circ} \mathrm{C}$ immediate coagulation of tissue is induced, with irreversible damage to the inner structure of the cells, using $100-110^{\circ} \mathrm{C}$, the tissue vaporizes and carbonizes [14]. At the beginning of the application of RFA on PDAC, high morbidity $(0-40 \%)$ and mortality $(0-25 \%)$ rates have been reported [15]. Later, ex vivo studies demonstrated that an adjustment of both temperature and length of the dispensed energy would conduce to better outcomes with fewer complications [16, 17]. Although several temperatures have been used, ranging from less than $30^{\circ} \mathrm{C}$ to $90^{\circ} \mathrm{C}$ according to the equipment used to perform RFA $[18,19]$, it seems that the ideal parameters to consider are actually represented by $90^{\circ} \mathrm{C}$ for 5 minutes, with a distance of 10 and $15 \mathrm{~mm}$ between probe and duodenum and portomesenteric axis, respectively [20]. The electrode must be introduced inside the tumor under ultrasound or CTguidance and the procedure can be monitored in real time by ultrasound with a safe distance of the RFA probe from duodenum or portomesenteric vessels of 5-10 mm (Figure 1). The procedure can either be performed through laparotomy, percutaneously, or through an endoscopic approach $[21,22]$. These mini-invasive techniques could be useful to avoid laparotomy, in patients unsuitable for surgery or in case of LAPC of the body-tail of the pancreas without symptoms.

2.2. IRE. IRE is a nonthermal technique that induces cell death. The ablative effect is based on the delivery of short high-voltage electric current fields that induce cell death. The application of short high-voltage electric pulses, conveyed by one or more monopolar electrodes, causes the irreversible permeabilization of the lipid bilayer, the disruption of intracellular homeostasis, and the activation of apoptotic pathways, ultimately resulting in cell death of neoplastic cells [30-36]. Interestingly, and differently from RFA, IRE is able to preserve surrounding structures, such as the underlying matrix that can work again as a scaffold for the healing tissue, or the vital structures like nerves or vessels [37-39]. Narayanan et al. in a retrospective review of 101 IRE procedures performed on different organs for tumors abutting or encasing major vessels reported a rate of vascular changes of only $4.4 \%$ (thrombosis or mild vascular narrowing phenomena) demonstrating a very high rate of patency of the major vessels in humans, after the application of IRE [40]. The proper ability of IRE to preserve the vessels could be a fundamental aspect when the tumor encases the major peripancreatic vessels, when the application of RFA could result as difficult, dangerous, and inefficacious (because of the heat-sink effect). However, it has been advocated that the cellular damage induced by IRE could be partially thermal. In fact, in some conditions of high intensity, current applied IRE can produce a coagulative necrosis similar to the one produced by thermal techniques [41]. Dunki-Jacobs et al. further investigated this aspect, concluding that IRE does not produce significant thermal energy, at least using the settings most commonly applied in clinical treatment. On the other side, they demonstrated that the presence of metallic stent could increase the risk of producing thermal injuries, because of the conductivity of the metal [42]. This aspect might be important in those patients carrying a biliary metallic stent for jaundice palliation. Hence, it should be kept in mind that IRE is not a "pure" nonthermal technique and that it remains connected in some way with thermal effects. Treatment planning of IRE is of utmost importance and several tools are available to properly manage the application of the technique [43-45]. Martin accurately described the procedure with the ideal settings on pancreas $[46,47]$.

\section{Indications and Contraindications}

Preoperative work-up should always include routine laboratory tests (including CA 19-9 levels) and a 3-phase CT-scan of the abdomen in order to assess exactly the location and the dimension of the tumor, the type of vascular infiltration, and the possible presence of abdominal metastases. Local ablative therapies, such as RFA and IRE, should be allotted to those tumors that show a local growth pattern without systemic involvement and should be considered as consolidative therapies in the multimodal therapeutic approach to LAPC. The decision to perform one or the other should be taken by a multidisciplinary group, considering the patients' comorbidities and quality of life, the natural history of the tumor, and, mostly, the response to medical oncological treatments. The assessment of resectability of LAPC after neoadjuvant therapy is still difficult [48]. In the FOLFIRINOX era, imaging seems to have no longer ability in determining the real response rate after neoadjuvant therapy [49]. In the future, RFA and IRE will be applied more often as "salvage" cytoreductive therapies or in the context of properly designed clinical trials, at least until randomized controlled trials will not demonstrate their oncological efficacy. Furthermore, it is of paramount importance that RFA and IRE should be performed selectively in high-volume HPB centers, and, for percutaneous-only approaches, by experienced interventional radiologists.

3.1. RFA. Indications are as follows. The most common worldwide application of RFA on PDAC is represented by the treatment of stage-III patients, either in case of no further response to standard systemic treatments or as an upfront option at the time of diagnosis [15, 16, 18, 28, 50-58]. However, some studies included also stage-IV metastatic patients 

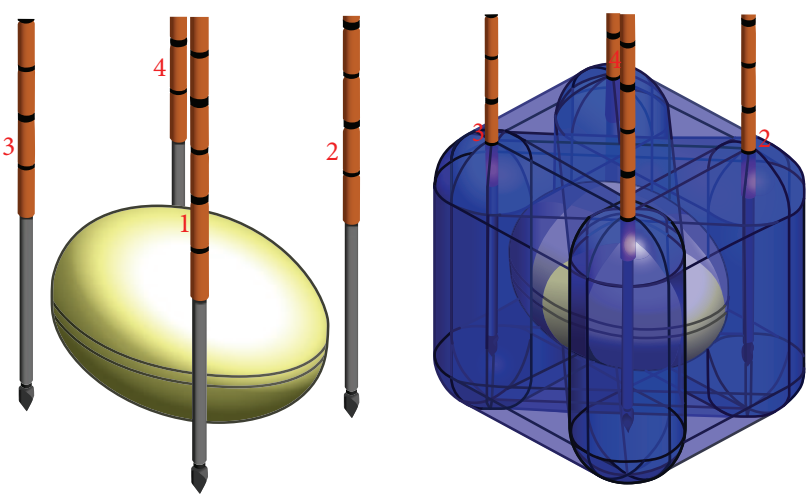

Figure 2: Example of a computerized model of the application of a 4-needle IRE technique. The yellow oval represents the tumor. Crossing blue beams represent the energy developed between each couple of probes.

$[18,19,59,60]$, probably to induce a positive modulation of the immune system [61]. Recently, the application of RFA upfront has been justified on the basis of a presumed immunological antitumoral stimulation aroused by RFA [50]; a randomized controlled study to prove or to disown it is currently running. However, RFA should be considered as a new tool in the surgeon's toolbox, in the context of a multidisciplinary approach to PDAC.

Tumor diameter is not a crucial parameter in the evaluation of the application of RFA as the technique itself allows ablating up to $5 \mathrm{~cm}$ or more [62]. Unfortunately, because of the proximity of vital structures surrounding PDAC (infiltrated by definition in LAPC), the whole ablation of the tumor would result in being too risky. Then, it is preferred to treat the biggest possible area, performing also pull-backs of the tip, leaving a "security ring" at the periphery of the tumor in order to avoid thermal injuries of the nearby structures [63]. This viable tissue at the periphery of the tumor will later be the target of the radiotherapy, to complete the ablation of the tumor [17].

Contraindications are as follows. RFA can interfere with implanted pacemakers and cardioverter defibrillators due to electromagnetic energy [64]. Hence, a cardiac evaluation is recommended in this special subset of patients, for a possible resynchronization of these devices.

3.2. IRE. Indications are as follows. Almost all the applications of IRE on PDAC are on stage-III LAPC [23, 24, 27, 47, 65-71]. Narayanan et al. reported three cases of application of IRE on stage-IV patients with centimetric liver metastases from PDAC and two cases of application of IRE as a "bridge" therapy in LAPC before submitting the patients successfully to a radical surgical resection [72]. Simultaneously, some papers report promising results on the use of IRE for margin accentuation, as a technique to reduce the rate of $\mathrm{R} 1$ resections in case of locally advanced/borderline resectable PDAC [24, $65,68,73]$. In general, IRE works better on tumor sizes that are 3 to $3.5 \mathrm{~cm}$ and it is important to plan the ablation technique properly (Figure 2) in order to treat the whole tumor [74]. In addition, the application of IRE seems to be more appropriate than RFA when the tumor encapsulates the superior mesenteric artery. In fact, the application of multiple needles allows bracketing the artery and treating. Furthermore, the negligible amount of heat associated with IRE allows safe and efficacious ablations.

Contraindications are as follows. In general, electric fields applied to human body can cause arrhythmias; hence, it is of utmost importance to reduce this risk synchronizing pulsing with the heart rhythm, using a dedicated device [75]. For these reasons, IRE is contraindicated in patients with pacemakers or with cardiac arrhythmias. Moreover, a metallic biliary stent should be removed intraoperatively before IRE, because the presence of the metal could increase the risk of thermal injury [70].

\section{Oncological Outcomes}

All the results regarding the oncological outcomes of the application of RFA and IRE on PDAC are biased by the nature itself of the studies. The reports include very heterogeneous populations of patients, with either stage-III or stage-IV disease. There are no randomized controlled studies available. Most of them were created as phase-I studies in order to demonstrate the safety of the techniques; then, oncological outcomes were only secondary goals. Despite these intrinsic problems, some encouraging results can be extracted.

4.1. RFA. Given that all patients treated with RFA will relentlessly progress $[16,53,57,60]$, some papers report good oncological results obtained with the use of RFA on PDAC. Spiliotis et al. reported a reassuring mean survival of 30 months for patients suffering from PDAC treated with RFA, compared to the 13 months' survival for patients receiving a standard systemic treatment $(p=0.0048)$ [18]. Giardino et al. cited a median overall survival (OS) for their whole series ( $n=107$ ) of 25.6 months, 14.7 months in the group of patients receiving RFA plus several possible systemic treatments, and 25.6 months in the group treated with primary treatments plus RFA plus further systemic treatments $(p=0.004)$. Interestingly, those patients who received this latter therapy, the so-called "triple approach strategy," with RFA plus radiochemotherapy plus intra-arterial chemotherapy with further systemic treatments, had an OS of 34.0 months [17].

4.2. IRE. Despite the increasing number of papers reporting the application of IRE on PDAC, none of these studies is designed to demonstrate the oncological efficacy of the procedure. In fact, they mostly deal with safety and feasibility issues and for this reason the populations considered are not ideal models for the analysis of oncological outcomes. Table 1 shows the studies reporting data on the efficacy of IRE; however, all these results must be considered cautiously. Interestingly, two papers described five cases of downstaging with R0-resections of LAPC treated with percutaneous IRE $[26,27]$.

A recent paper from Martin et al. reports an outstanding median OS of 24.9 months (range 12.4-85 months; 
TABLE 1: Efficacy of IRE on PDAC.

\begin{tabular}{|c|c|c|c|c|}
\hline Author & $\begin{array}{c}\text { Number of } \\
\text { patients }\end{array}$ & Approach & Type of study & $\begin{array}{c}\text { Survival } \\
\text { (mo.) }\end{array}$ \\
\hline Martin et al. [23] & 54 & $\begin{array}{c}\text { Open (52) } \\
\text { Percutaneously (2) }\end{array}$ & $\begin{array}{l}\text { Propensity-matched } \\
\text { comparison with } \\
\text { standard chemo- or } \\
\text { chemoradiation }\end{array}$ & 20.2 \\
\hline Martin et al. [24] & 200 & Open & $\begin{array}{c}\text { Data from } \\
\text { multicenter registry }\end{array}$ & 24.9 \\
\hline $\begin{array}{l}\text { Trueba-Arguiñarena } \\
\text { et al. [25] }\end{array}$ & 1 & Percutaneously & Case report & f-up 12 mo. \\
\hline Narayanan et al. [26] & 43 & Percutaneously & Prospective & 16.2 \\
\hline Belfiore et al. [27] & 20 & Percutaneously & Retrospective & 12.9 \\
\hline Pai et al. [28] & 5 & Percutaneously & $\begin{array}{c}\text { Phase-1 safety and } \\
\text { feasibility }\end{array}$ & $\begin{array}{c}\text { Range } \\
1-6 \text { mo. }\end{array}$ \\
\hline Paiella et al. [29] & 10 & Open & $\begin{array}{l}\text { Phase- } 1 \text { safety and } \\
\text { feasibility }\end{array}$ & $\begin{array}{c}\text { Median 6.4, } \\
\text { range } \\
2.9-15.9 \\
\end{array}$ \\
\hline
\end{tabular}

$n=200$ ), for patients treated with IRE in situ or pancreatic resections with major vascular resections and IRE for the margin accentuation, after 6 months (median) of induction chemotherapy or chemo(radio)therapy [24]. As the authors state in the paper, the population considered is made of highly selected patients and this represents an important selection bias. However, these results are very surprising and encouraging, especially if compared with the historical populations of patients reported in literature suffering from LAPC.

Recently, Philips et al. reported an increased risk of accelerating the tumor growth after the application of incomplete sessions of IRE in a murine model. This worrisome finding should be further clarified and possibly verified in clinical scenarios [76].

\section{Complications}

The majority of the complications caused by local ablative techniques are consequence of an uncontrolled heating of the structures surrounding the tumor, rather than a direct lesion caused by the tip of the probe used. Therefore, obviously, it is of paramount importance to plan properly the procedure, setting the parameters according to location, dimensions, and morphology of the tumor.

5.1. RFA. The first clinical applications of RFA were afflicted by a high rate of morbidity and mortality, ranging from 0 to $40 \%$ and from 0 to $25 \%$, respectively [15]. Once the temperature was lowered from 105 to $90^{\circ} \mathrm{C}$ for 5 minutes' length, the reported number of complications reduced in parallel [16, 17]. The deaths related to RFA were most commonly caused by gastrointestinal hemorrhages. The most recent cohort of patients treated with RFA comes again from Girelli et al. They reported a reduction of the morbidity rate to $8 \%$, with a mortality rate of $0 \%$ [50]. The overall reported rates of RFA-related complications and RFA-related mortality are 13.6 and $1.5 \%$, respectively [13]. The most common complications reported in literature are gastrointestinal hemorrhages and minor local bleedings, acute pancreatitis (mild or severe), pancreatic and biliary fistulas, duodenal injury (thermal or direct), and portal vein thrombosis. It is suggested to cool the duodenum during the procedure with a cold saline solution administered using the nasogastric tube, to preserve it from the possible thermal injury [20].

5.2. IRE. A recent systematic review reported an IRE-related complication rate of $13 \%$, with an IRE-related mortality of $2 \%$ [13]. The overall reported complications rate of the percutaneous approach is $29 \%$ [77]. Martin et al., in a recent study with a population of 200 patients suffering from LAPC treated with IRE, showed an overall rate of adverse events of 37\% (74 patients with 149 overall complications) and a mortality rate of $2 \%$ [24]. The largest single-center percutaneous series of 50 IRE described an overall number of 27 complications [26]. The most common complications (including both percutaneous and open techniques) described after the use of IRE on pancreas are pancreatitis, pneumothorax, hematoma, abdominal pain, bile leakage, pancreatic leakage, duodenal leakage, duodenal ulcer, and deep vein thrombosis.

\section{Ablative Techniques and Imaging}

One of the most interesting and useful aspects of the application of the ablative techniques on PDAC is the possibility to appraise the amount of tissue ablated and the relationship between the treated area and tumor margins.

6.1. RFA. For RFA, and in general for "thermal techniques," the gold standard of imaging is represented by cross-sectional imaging via helical CT-scan, rather than ultrasonography [78]. A postablative hypointense area can be observed as result of the treatment (Figure 3). At our institution, we perform a three-phase contrast-enhanced CT-scan of the 


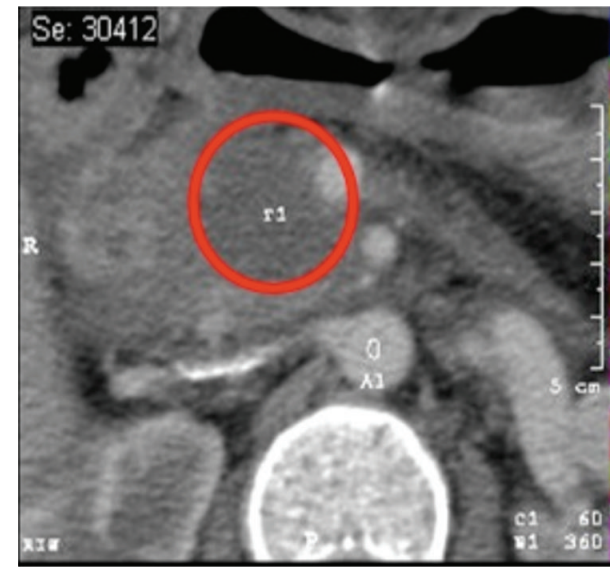

(a)

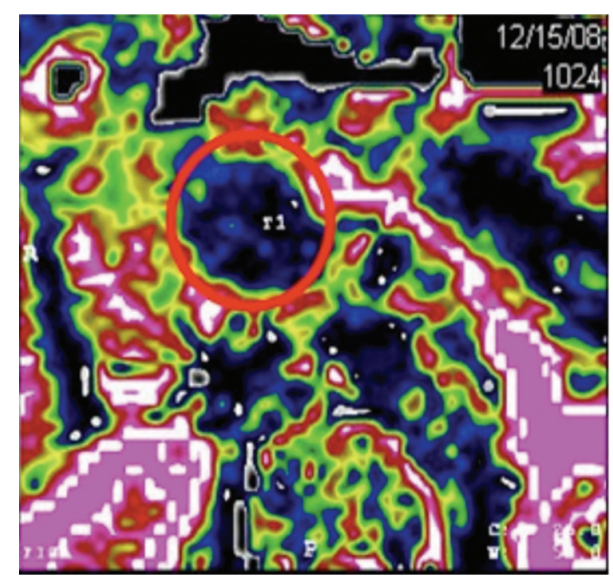

(b)

FIgure 3: (a) Preoperative CT-scan of a locally advanced pancreatic cancer. (b) Post-RFA perfusion CT-scan, showing a postablative area of decreased perfusion within the head of the pancreas. Copyright Chirurgia del Pancreas Verona.

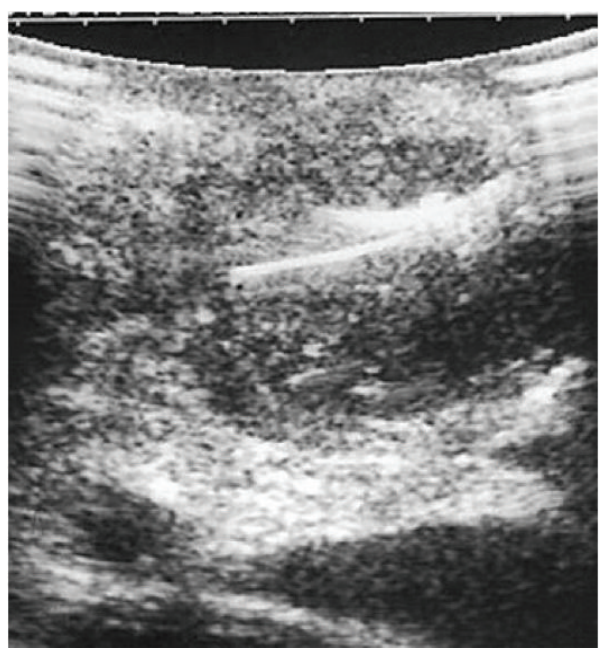

(a)

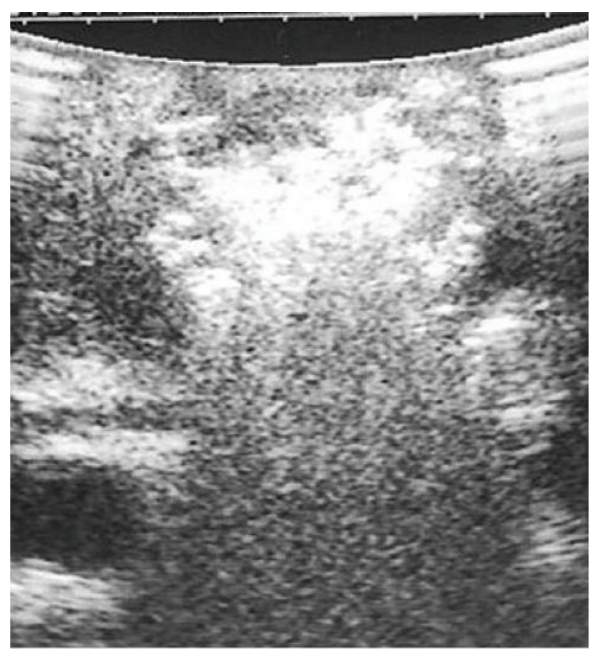

(b)

FIGURE 4: (a) The tip for RFA is placed inside the tumor under US-guidance. (b) During RFA, the lesion becomes immediately hyperechoic.

abdomen at postoperative days 7 and 30. During the procedure, ultrasonography can both guide the tip and detect the immediate results of the thermal damage (Figure 4).

6.2. IRE. Because of the nonthermal noncoagulative action of IRE and because of the consequent preservation of the vessels, the application of contrast-enhanced CT-scan after IRE would not have the same results as for RFA. Several techniques have been used to evaluate the effect of the application of IRE. Magnetic resonance electrical impedance tomography (MREIT) seems to be able to identify the areas with insufficient electric field, in order to label potential untreated zones after IRE [79]. Either contrast-enhanced or diffusion weighted MRI seems to be able to depict the tissue zones ablated with IRE [80-82]. Even if using a swine model, a recent study stated that the best identification of tissue ablation after IRE is obtained with portal vein phase CT-scan. Anyway, differently from RFA, during CT-scan, a contrast enhancement can be appreciated on the more delayed venous phase, due to the congestion of blood in the tumor vessels $[32,83,84]$. Ultrasound findings during and after IRE could be useful to evaluate the approximate area of ablation. In the acute phase, a hypoechoic area can be registered, with a hyperechoic external rim that forms 90-120 minutes after the treatment $[85,86]$. However, Martin et al. state that an early postoperative scanning after IRE should be performed only to rule out possible complications (deep vein thrombosis) and not to evaluate ablation efficacy [24]. It still has to be evaluated if a serum CA 19-9 level decrease could be used as predictor of efficacy. 


\section{Ablative Techniques and Immune System}

The strongest factor supporting the clinical application of the ablative techniques, especially of RFA, is represented by their positive antitumoral effect on immune system. Nowadays, thanks to the several studies that have been published, RFA is called prudently "endogenous vaccine" for PDAC. How strong is this power and which are the best timing and proper methods to use it remain to be established.

7.1. RFA. All the processes involving the modulation of immune system have been exhaustively described by Chu and Dupuy [87]. While the direct effect of RFA is clearly represented by the necrotic area immediately identifiable after the procedure, on the other hand, the indirect effects are on the viable zone adjacent to this area (transition or peripheral zone). The cells populating the peripheral zone are affected by the RFA in terms of alteration of metabolic endocellular processes that makes them quite sensible to further cytolytic therapies, such as chemo- or chemoradiotherapy. These effects result, ultimately, in the almost total destruction of the tumor. In parallel to this "local" action, RFA can cause a "systemic" immune response involving proinflammatory cytokines [88-90], lymphocytes (T-, B-, and NK-types) [9194], and antibodies [95] that are responsible for acquired antitumoral antigen-specific immunity $[96,97]$ that could confer better survival in some patients treated with RFA. It also seems promising to use the synergic use of RFA together with topic specific cytolytic agents or with immunotherapy with monoclonal antibodies or vaccine [98]. However, most of the findings described come from experimental models or from in vivo results from organs other than pancreas. Of course, there is need for more preclinical models, investigational studies, and large randomized controlled clinical trials to demonstrate the effects of RFA on PDAC selectively.

7.2. IRE. The immune system involvement after IRE has not been thoroughly investigated yet.

Some reports support the evidence that since the proteins are not denatured in IRE (differently from in RFA), in theory, this could result in a weak specific antigenic stimulation against the tumor. In fact, Al-Sakere et al., using murine models of sarcoma treated with IRE, showed that there is no local infiltration of tumor cells among the treated tissue. An early and prolonged decrease in both $\mathrm{T}$ lymphocytes (both CD4+ and CD8+) and antigen presenting cells can be detected within a couple of hours after IRE [99]. As they support, this is a demonstration that IRE does not need the involvement of the immune system to kill neoplastic cells and, for this reason, it could be applied on immunosuppressed patients too. On the other hand, other reports reached the evidence that both local and systemic immune antitumoral stimulation are enhanced after IRE [100, 101]. This aspect could be referred to the peculiar type of cellular death caused by IRE: the activation of the apoptotic processes leads to the release of intact and stimulating endogenous tumoral antigens able to induce a strong global antitumoral activity. Ultimately, according to Neal II et al., IRE would be able to generate the "three signals' sequence" that is mandatory for the production of a cytotoxic T-cell response [101].

These conflicting reports demonstrate how far we are from the understanding of the exact involvement of the immune system and how much we need further preclinical and clinical models.

\section{Conclusions}

RFA and IRE represent an innovation on the multimodal treatment of LAPC. The undeniable advantages connected with the use of these techniques are represented by low morbidity, reduced costs, possible percutaneous application, almost selective action with preservation of peritumoral tissues, possible application to patients at a high-risk for surgery, and suspected positive immune stimulation. Moreover, taking into account their positive effect on the immune system, they could be potentially very useful in those patients that, somehow, show an indolent disease, with a prevalent local growth and without a wide systemic involvement.

Nevertheless, as for any other technology introduced in medical practice, RFA and IRE have to be evaluated prospectively and systematically according to the IDEAL framework for evaluation of surgical innovation [102]. In the IDEAL paradigm for the introduction of new technologies in surgery, the application of RFA and IRE on LAPC is still stuck on the 2a phase where few people still adopt the technique, where the patients are selected, where the outcomes are mostly safety and feasibility, and where the clinical outcomes are timidly reported. Hence, the current available evidence is still not sufficient to permit conclusions about long-term benefits.

Nowadays, the patients suffering from LAPC are still waiting for answers that medical oncologists cannot give. Surgery and new ablative technologies can play an important role in giving hope, prolonging survival, and improving quality of life of the patients suffering from LAPC. However, we must move toward a rigorous evaluation of these new procedures through the creation of appropriate randomized controlled studies.

\section{Conflict of Interests}

The authors declare that there is no conflict of interests regarding the publication of this paper.

\section{References}

[1] American Cancer Society, Cancer Facts \& Figures 2013, American Cancer Society, 2013.

[2] H. G. Smeenk, T. C. K. Tran, J. Erdmann, C. H. J. van Eijck, and J. Jeekel, "Survival after surgical management of pancreatic adenocarcinoma: does curative and radical surgery truly exist?" Langenbeck's Archives of Surgery, vol. 390, no. 2, pp. 94-103, 2005.

[3] J. Kleeff, C. Reiser, U. Hinz et al., "Surgery for recurrent pancreatic ductal adenocarcinoma," Annals of Surgery, vol. 245, no. 4, pp. 566-572, 2007. 
[4] A. G. Morganti, M. Massaccesi, G. La Torre et al., "A systematic review of resectability and survival after concurrent chemoradiation in primarily unresectable pancreatic cancer," Annals of Surgical Oncology, vol. 17, no. 1, pp. 194-205, 2010.

[5] H. Oettle, S. Post, P. Neuhaus et al., "Adjuvant chemotherapy with gemcitabine vs observation in patients undergoing curative-intent resection of pancreatic cancer: a randomized controlled trial," The Journal of the American Medical Association, vol. 297, no. 3, pp. 267-277, 2007.

[6] J. P. Neoptolemos, D. D. Stocken, H. Friess et al., "A randomized trial of chemoradiotherapy and chemotherapy after resection of pancreatic cancer," The New England Journal of Medicine, vol. 350, no. 12, pp. 1200-1210, 2004.

[7] W. F. Regine, K. A. Winter, R. A. Abrams et al., "Fluorouracil vs gemcitabine chemotherapy before and after fluorouracilbased chemoradiation following resection of pancreatic adenocarcinoma: a randomized controlled trial," The Journal of the American Medical Association, vol. 299, no. 9, pp. 1019-1026, 2008.

[8] C. M. R. Lima, M. R. Green, R. Rotche et al., "Irinotecan plus gemcitabine results in no survival advantage compared with gemcitabine monotherapy in patients with locally advanced or metastatic pancreatic cancer despite increased tumor response rate," Journal of Clinical Oncology, vol. 22, no. 18, pp. 3776-3783, 2004.

[9] E. Poplin, Y. Feng, J. Berlin et al., "Phase III, randomized study of gemcitabine and oxaliplatin versus gemcitabine (fixeddose rate infusion) compared with gemcitabine (30-minute infusion) in patients with pancreatic carcinoma E6201: a trial of the Eastern Cooperative Oncology Group," Journal of Clinical Oncology, vol. 27, no. 23, pp. 3778-3785, 2009.

[10] C. Louvet, R. Labianca, P. Hammel et al., "Gemcitabine in combination with oxaliplatin compared with gemcitabine alone in locally advanced or metastatic pancreatic cancer: results of a GERCOR and GISCAD phase III trial," Journal of Clinical Oncology, vol. 23, no. 15, pp. 3509-3516, 2005.

[11] V. Heinemann, M. Haas, and S. Boeck, "Neoadjuvant treatment of borderline resectable and non-resectable pancreatic cancer," Annals of Oncology, vol. 24, no. 10, pp. 2484-2492, 2013.

[12] F. Huguet, N. Girard, C. S. Guerche, C. Hennequin, F. Mornex, and D. Azria, "Chemoradiotherapy in the management of locally advanced pancreatic carcinoma: a qualitative systematic review," Journal of Clinical Oncology, vol. 27, no. 13, pp. 22692277, 2009.

[13] S. J. E. Rombouts, J. A. Vogel, H. C. van Santvoort et al., "Systematic review of innovative ablative therapies for the treatment of locally advanced pancreatic cancer," British Journal of Surgery, vol. 102, no. 3, pp. 182-193, 2015.

[14] E. M. W. Sonnenberg and L. Solbiati, Tumor Ablation, Springer, New York, NY, USA, 2005.

[15] M. G. Keane, K. Bramis, S. P. Pereira, and G. K. Fusai, "Systematic review of novel ablative methods in locally advanced pancreatic cancer," World Journal of Gastroenterology, vol. 20, no. 9, pp. 2267-2278, 2014.

[16] R. Girelli, I. Frigerio, A. Giardino et al., "Results of 100 pancreatic radiofrequency ablations in the context of a multimodal strategy for stage III ductal adenocarcinoma," Langenbeck's Archives of Surgery, vol. 398, no. 1, pp. 63-69, 2013.

[17] A. Giardino, R. Girelli, I. Frigerio et al., "Triple approach strategy for patients with locally advanced pancreatic carcinoma," $H P B$, vol. 15, no. 8, pp. 623-627, 2013.
[18] J. D. Spiliotis, A. C. Datsis, N. V. Michalopoulos et al., "Radiofrequency ablation combined with palliative surgery may prolong survival of patients with advanced cancer of the pancreas," Langenbeck's Archives of Surgery, vol. 392, no. 1, pp. 55-60, 2007.

[19] Y. Wu, Z. Tang, H. Fang et al., "High operative risk of cooltip radiofrequency ablation for unresectable pancreatic head cancer 1," Journal of Surgical Oncology, vol. 94, no. 5, pp. 392395, 2006.

[20] S. Fegrachi, I. Q. Molenaar, J. H. Klaessens, M. G. Besselink, J. A. Offerhaus, and R. van Hillegersberg, "Radiofrequency ablation of the pancreas with and without intraluminal duodenal cooling in a porcine model," The Journal of Surgical Research, vol. 184, no. 2, pp. 867-872, 2013.

[21] S. Rossi, F. T. Viera, G. Ghittoni et al., "Radiofrequency ablation of pancreatic neuroendocrine tumors: a pilot study of feasibility, efficacy, and safety," Pancreas, vol. 43, no. 6, pp. 938-945, 2014.

[22] T. J. Song, D. W. Seo, S. Lakhtakia et al., "Initial experience of EUS-guided radiofrequency ablation of unresectable pancreatic cancer," Gastrointestinal Endoscopy, vol. 83, no. 2, pp. 440-443, 2016.

[23] R. C. G. Martin II, K. McFarland, S. Ellis, and V. Velanovich, "Irreversible electroporation in locally advanced pancreatic cancer: potential improved overall survival," Annals of Surgical Oncology, vol. 20, supplement 3, pp. S443-S449, 2013.

[24] R. C. Martin, D. Kwon, S. Chalikonda et al., "Treatment of 200 locally advanced (stage III) pancreatic adenocarcinoma patients with irreversible electroporation: safety and efficacy," Annals of Surgery, vol. 262, no. 3, pp. 486-494, 2015.

[25] F. J. Trueba-Arguiñarena, D. S. de Prado-Otero, and R. PovesAlvarez, "Pancreatic adenocarcinoma treated with irreversible electroporation case report: first experience and outcome," Medicine, vol. 94, no. 26, p. e946, 2015.

[26] G. Narayanan, P. J. Hosein, C. M. S. R. Lima et al., "Percutaneous irreversible electroporation (IRE) in the management of pancreatic cancer," Journal of Clinical Oncology, vol. 32, supplement, abstract e15249, 2014.

[27] M. P. Belfiore, F. M. Ronza, F. Romano et al., "Percutaneous CTguided irreversible electroporation followed by chemotherapy as a novel neoadjuvant protocol in locally advanced pancreatic cancer: our preliminary experience," International Journal of Surgery, vol. 21, supplement 1, pp. S34-S39, 2015.

[28] M. Pai, J. Yang, X. Zhang et al., "PWE-055 Endoscopic ultrasound guided radiofrequency ablation (EUS-RFA) for pancreatic ductal adenocarcinoma," Gut, vol. 62, supplement 1, pp. A153-A153, 2013.

[29] S. Paiella, G. Butturini, I. Frigerio et al., "Safety and feasibility of Irreversible Electroporation (IRE) in patients with locally advanced pancreatic cancer: results of a prospective study," Digestive Surgery, vol. 32, no. 2, pp. 90-97, 2015.

[30] B. Rubinsky, G. Onik, and P. Mikus, "Irreversible electroporation: a new ablation modality-clinical implications," Technology in Cancer Research \& Treatment, vol. 6, no. 1, pp. 37-48, 2007.

[31] R. V. Davalos, L. M. Mir, and B. Rubinsky, "Tissue ablation with irreversible electroporation," Annals of Biomedical Engineering, vol. 33, no. 2, pp. 223-231, 2005.

[32] B. Al-Sakere, F. André, C. Bernat et al., "Tumor ablation with irreversible electroporation," PLOS ONE, vol. 2, no. 11, Article ID e1135, 2007.

[33] Z. Zhang, W. Li, D. Procissi, P. Tyler, R. A. Omary, and A. C. Larson, "Rapid dramatic alterations to the tumor microstructure in pancreatic cancer following irreversible electroporation ablation," Nanomedicine, vol. 9, no. 8, pp. 1181-1192, 2014. 
[34] E. W. Lee, D. Wong, S. V. Prikhodko et al., "Electron microscopic demonstration and evaluation of irreversible electroporation-induced nanopores on hepatocyte membranes," Journal of Vascular and Interventional Radiology, vol. 23, no. 1, pp. 107113, 2012.

[35] M. L. Yarmush, A. Golberg, G. Serša, T. Kotnik, and D. Miklavčič, "Electroporation-based technologies for medicine: principles, applications, and challenges," Annual Review of Biomedical Engineering, vol. 16, pp. 295-320, 2014.

[36] A. Golberg and M. L. Yarmush, "Nonthermal irreversible electroporation: fundamentals, applications, and challenges," IEEE Transactions on Biomedical Engineering, vol. 60, no. 3, pp. 707-714, 2013.

[37] E. Maor, A. Ivorra, J. Leor, and B. Rubinsky, "Irreversible electroporation attenuates neointimal formation after angioplasty," IEEE Transactions on Biomedical Engineering, vol. 55, no. 9, pp. 2268-2274, 2008.

[38] E. Maor, A. Ivorra, and B. Rubinsky, "Intravascular irreversible electroporation: theoretical and experimental feasibility study," in Proceedings of the 30th Annual International Conference of the IEEE Engineering in Medicine and Biology Society (EMBS '08), pp. 2051-2054, Vancouver, Canada, August 2008.

[39] H. Schoellnast, S. Monette, P. C. Ezell et al., "Acute and subacute effects of irreversible electroporation on nerves: experimental study in a pig model," Radiology, vol. 260, no. 2, pp. 421-427, 2011.

[40] G. Narayanan, S. Bhatia, A. Echenique, R. Suthar, K. Barbery, and J. Yrizarry, "Vessel patency post irreversible electroporation," CardioVascular and Interventional Radiology, vol. 37, no. 6, pp. 1523-1529, 2014.

[41] M. Faroja, M. Ahmed, L. Appelbaum et al., "Irreversible electroporation ablation: is all the damage nonthermal?" Radiology, vol. 266, no. 2, pp. 462-470, 2013.

[42] E. M. Dunki-Jacobs, P. Philips, and R. C. G. Martin II, "Evaluation of thermal injury to liver, pancreas and kidney during irreversible electroporation in an in vivo experimental model," The British Journal of Surgery, vol. 101, no. 9, pp. 1113-1121, 2014.

[43] M. Marčan, D. Pavliha, B. Kos, T. Forjanič, and D. Miklavčič, "Web-based tool for visualization of electric field distribution in deep-seated body structures and planning of electroporationbased treatments," BioMedical Engineering OnLine, vol. 14, supplement 3, article S4, 2015.

[44] A. Zupanic, B. Kos, and D. Miklavcic, "Treatment planning of electroporation-based medical interventions: electrochemotherapy, gene electrotransfer and irreversible electroporation," Physics in Medicine and Biology, vol. 57, no. 17, pp. 5425-5440, 2012.

[45] D. Miklavcic and R. V. Davalos, "Electrochemotherapy (ECT) and irreversible electroporation (IRE)-advanced techniques for treating deep-seated tumors based on electroporation," BioMedical Engineering OnLine, vol. 14, supplement 3, article I1, 2015.

[46] R. C. G. Martin, "Irreversible electroporation of locally advanced pancreatic head adenocarcinoma," Journal of Gastrointestinal Surgery, vol. 17, no. 10, pp. 1850-1856, 2013.

[47] R. C. G. Martin, P. Philips, S. Ellis, D. Hayes, and S. Bagla, "Irreversible electroporation of unresectable soft tissue tumors with vascular invasion: effective palliation," BMC Cancer, vol. 14, article 540, 2014.

[48] C. Tosolini, C. W. Michalski, and J. Kleeff, "Response evaluation following neoadjuvant treatment of pancreatic cancer patients,"
World Journal of Gastrointestinal Surgery, vol. 5, no. 2, pp. 12-15, 2013.

[49] C. R. Ferrone, G. Marchegiani, T. S. Hong et al., "Radiological and surgical implications of neoadjuvant treatment with FOLFIRINOX for locally advanced and borderline resectable pancreatic cancer," Annals of Surgery, vol. 261, no. 1, pp. 12-17, 2015.

[50] I. Frigerio, R. Girelli, A. Giardino, P. Regi, R. Salvia, and C. Bassi, "Short term chemotherapy followed by radiofrequency ablation in stage III pancreatic cancer: results from a single center," Journal of Hepato-Biliary-Pancreatic Sciences, vol. 20, no. 6, pp. 574-577, 2013.

[51] R. Girelli, I. Frigerio, R. Salvia, E. Barbi, P. Tinazzi Martini, and C. Bassi, "Feasibility and safety of radiofrequency ablation for locally advanced pancreatic cancer," British Journal of Surgery, vol. 97, no. 2, pp. 220-225, 2010.

[52] M. Cantore, R. Girelli, A. Mambrini et al., "Combined modality treatment for patients with locally advanced pancreatic adenocarcinoma," The British Journal of Surgery, vol. 99, no. 8, pp. 1083-1088, 2012.

[53] A. K. Siriwardena, "Radiofrequency ablation for locally advanced cancer of the pancreas," Journal of the Pancreas, vol. 7, no. 1, pp. 1-4, 2006.

[54] A. W. Steel, A. J. Postgate, S. Khorsandi et al., "Endoscopically applied radiofrequency ablation appears to be safe in the treatment of malignant biliary obstruction," Gastrointestinal Endoscopy, vol. 73, no. 1, pp. 149-153, 2011.

[55] P. Hadjicostas, N. Malakounides, C. Varianos, E. Kitiris, F. Lerni, and P. Symeonides, "Radiofrequency ablation in pancreatic cancer," $H P B$, vol. 8, no. 1, pp. 61-64, 2006.

[56] P. Figueroa-Barojas, M. R. Bakhru, N. A. Habib et al., "Safety and efficacy of radiofrequency ablation in the management of unresectable bile duct and pancreatic cancer: a novel palliation technique," Journal of Oncology, vol. 2013, Article ID 910897, 5 pages, 2013.

[57] S. Varshney, A. Sewkani, S. Sharma et al., "Radiofrequency ablation of unresectable pancreatic carcinoma: feasibility, efficacy and safety," Journal of the Pancreas, vol. 7, no. 1, pp. 74-78, 2006.

[58] R. Casadei, C. Ricci, R. Pezzilli et al., "A prospective study on radiofrequency ablation locally advanced pancreatic cancer," Hepatobiliary \& Pancreatic Diseases International, vol. 9, no. 3, pp. 306-311, 2010.

[59] Y. Matsui, A. Nakagawa, Y. Kamiyama, K. Yamamoto, N. Kubo, and Y. Nakase, "Selective thermocoagulation of unresectable pancreatic cancers by using radiofrequency capacitive heating," Pancreas, vol. 20, no. 1, pp. 14-20, 2000.

[60] R. S. Date and A. K. Siriwardena, "Radiofrequency ablation of the pancreas. II: intra-operative ablation of non-resectable pancreatic cancer. A description of technique and initial outcome," Journal of the Pancreas, vol. 6, no. 6, pp. 588-592, 2005.

[61] R. Waitz and S. B. Solomon, "Can local radiofrequency ablation of tumors generate systemic immunity against metastatic disease?" Radiology, vol. 251, no. 1, pp. 1-2, 2009.

[62] L. Tiong and G. J. Maddern, "Systematic review and metaanalysis of survival and disease recurrence after radiofrequency ablation for hepatocellular carcinoma," The British Journal of Surgery, vol. 98, no. 9, pp. 1210-1224, 2011.

[63] M. D’Onofrio, G. Zamboni, N. Faccioli, P. Capelli, and R. Pozzi Mucelli, "Ultrasonography of the pancreas. 4. Contrastenhanced imaging," Abdominal Imaging, vol. 32, no. 2, pp. 171181, 2007. 
[64] M. W. Sweesy, J. L. Holland, and K. W. Smith, "Electromagnetic interference in cardiac rhythm management devices," AACN Clinical Issues, vol. 15, no. 3, pp. 391-403, 2004.

[65] R. C. G. Martin II, K. McFarland, S. Ellis, and V. Velanovich, "Irreversible electroporation therapy in the management of locally advanced pancreatic adenocarcinoma," Journal of the American College of Surgeons, vol. 215, no. 3, pp. 361-369, 2012.

[66] S. Bagla and D. Papadouris, "Percutaneous irreversible electroporation of surgically unresectable pancreatic cancer: a case report," Journal of Vascular and Interventional Radiology, vol. 23, no. 1, pp. 142-145, 2012.

[67] C. Mansson, M. Bergenfeldt, R. Brahmstaedt, B.-M. Karlson, P. Nygren, and A. Nilsson, "Safety and preliminary efficacy of ultrasound-guided percutaneous irreversible electroporation for treatment of localized pancreatic cancer," Anticancer Research, vol. 34, no. 1, pp. 289-293, 2014.

[68] M. J. Weiss and C. L. Wolfgang, "Irreversible electroporation: a novel pancreatic cancer therapy," Current Problems in Cancer, vol. 37, no. 5, pp. 262-265, 2013.

[69] P. Philips, D. Hays, and R. C. G. Martin, "Irreversible electroporation ablation (IRE) of unresectable soft tissue tumors: learning curve evaluation in the first 150 patients treated," PLoS ONE, vol. 8, no. 11, Article ID e76260, 2013.

[70] E. M. Dunki-Jacobs, P. Philips, and R. C. G. Martin II, "Evaluation of resistance as a measure of successful tumor ablation during irreversible electroporation of the pancreas," Journal of the American College of Surgeons, vol. 218, no. 2, pp. 179-187, 2014.

[71] S. Venkat, P. J. Hosein, and G. Narayanan, "Percutaneous approach to irreversible electroporation of the pancreas: Miami protocol," Techniques in Vascular and Interventional Radiology, vol. 18, no. 3, pp. 153-158, 2015.

[72] G. Narayanan, P. J. Hosein, G. Arora et al., "Percutaneous irreversible electroporation for downstaging and control of unresectable pancreatic adenocarcinoma," Journal of Vascular and Interventional Radiology, vol. 23, no. 12, pp. 1613-1621, 2012.

[73] D. Kwon, K. McFarland, V. Velanovich, and R. C. G. Martin II, "Borderline and locally advanced pancreatic adenocarcinoma margin accentuation with intraoperative irreversible electroporation," Surgery, vol. 156, no. 4, pp. 910-922, 2014.

[74] G. Narayanan, "Irreversible electroporation for treatment of liver cancer," Gastroenterology \& Hepatology, vol. 7, no. 5, pp. 313-316, 2011.

[75] A. Deodhar, T. Dickfeld, G. W. Single et al., "Irreversible electroporation near the heart: ventricular arrhythmias can be prevented with ECG synchronization," American Journal of Roentgenology, vol. 196, no. 3, pp. W330-W335, 2011.

[76] P. Philips, Y. Li, S. Li, C. R. St Hill, and R. C. Martin, "Efficacy of irreversible electroporation in human pancreatic adenocarcinoma: advanced murine model," Molecular Therapy-Methods \& Clinical Development, vol. 2, Article ID 15001, 2015.

[77] H. J. Scheffer, K. Nielsen, M. C. de Jong et al., "Irreversible electroporation for nonthermal tumor ablation in the clinical setting: a systematic review of safety and efficacy," Journal of Vascular and Interventional Radiology, vol. 25, no. 7, pp. 9971011, 2014

[78] S. S. Raman, D. S. K. Lu, D. J. Vodopich, J. Sayre, and C. Lassman, "Creation of radiofrequency lesions in a porcine model: correlation with sonography, CT, and histopathology," American Journal of Roentgenology, vol. 175, no. 5, pp. 1253$1258,2000$.
[79] M. Kranjc, F. Bajd, I. Sersa, E. J. Woo, and D. Miklavcic, "Ex vivo and in silico feasibility study of monitoring electric field distribution in tissue during electroporation based treatments," PLoS ONE, vol. 7, no. 9, Article ID e45737, 2012.

[80] F. Mahmood, R. H. Hansen, B. Agerholm-Larsen, K. S. Jensen, H. K. Iversen, and J. Gehl, "Diffusion-weighted MRI for verification of electroporation-based treatments," Journal of Membrane Biology, vol. 240, no. 3, pp. 131-138, 2011.

[81] Y. Guo, Y. Zhang, G. M. Nijm et al., "Irreversible electroporation in the liver: contrast-enhanced inversion-recovery MR imaging approaches to differentiate reversibly electroporated penumbra from irreversibly electroporated ablation zones," Radiology, vol. 258, no. 2, pp. 461-468, 2011.

[82] M. Kranjc, B. Markelc, F. Bajd et al., "In situ monitoring of electric field distribution in mouse tumor during electroporation," Radiology, vol. 274, no. 1, pp. 115-123, 2015.

[83] J. F. Edd, L. Horowitz, R. V. Davalos, L. M. Mir, and B. Rubinsky, "In vivo results of a new focal tissue ablation technique: irreversible electroporation," IEEE Transactions on Biomedical Engineering, vol. 53, no. 7, pp. 1409-1415, 2006.

[84] Y. J. Lee, D. S. K. Lu, F. Osuagwu, and C. Lassman, "Irreversible electroporation in porcine liver: acute computed tomography appearance of ablation zone with histopathologic correlation," Journal of Computer Assisted Tomography, vol. 37, no. 2, pp. 154158, 2013.

[85] L. Appelbaum, E. Ben-David, J. Sosna, Y. Nissenbaum, and S. N. Goldberg, "US findings after irreversible electroporation ablation: radiologic-pathologic correlation," Radiology, vol. 262, no. 1, pp. 117-125, 2012.

[86] C. R. Schmidt, P. Shires, and M. Mootoo, "Real-time ultrasound imaging of irreversible electroporation in a porcine liver model adequately characterizes the zone of cellular necrosis," $H P B$, vol. 14, no. 2, pp. 98-102, 2012.

[87] K. F. Chu and D. E. Dupuy, “Thermal ablation of tumours: biological mechanisms and advances in therapy," Nature Reviews Cancer, vol. 14, no. 3, pp. 199-208, 2014.

[88] M. Y. Ali, C. F. Grimm, M. Ritter et al., "Activation of dendritic cells by local ablation of hepatocellular carcinoma," Journal of Hepatology, vol. 43, no. 5, pp. 817-822, 2005.

[89] S. Evrard, C. Menetrier-Caux, C. Biota et al., "Cytokines pattern after surgical radiofrequency ablation of liver colorectal metastases," Gastroentérologie Clinique et Biologique, vol. 31, no. 2, pp. 141-145, 2007.

[90] A. M. Fietta, M. Morosini, I. Passadore et al., "Systemic inflammatory response and downmodulation of peripheral CD $25^{+}$Foxp $^{+}$T-regulatory cells in patients undergoing radiofrequency thermal ablation for lung cancer," Human Immunology, vol. 70, no. 7, pp. 477-486, 2009.

[91] M. C. Jansen, S. van Wanrooy, R. van Hillegersberg et al., "Assessment of systemic inflammatory response (SIR) in patients undergoing radiofrequency ablation or partial liver resection for liver tumors," European Journal of Surgical Oncology, vol. 34, no. 6, pp. 662-667, 2008.

[92] A. Zerbini, M. Pilli, D. Laccabue et al., "Radiofrequency thermal ablation for hepatocellular carcinoma stimulates autologous NK-cell response," Gastroenterology, vol. 138, no. 5, pp. 1931.e21942.e2, 2010.

[93] J. Hänsler, T. T. Wissniowski, D. Schuppan et al., "Activation and dramatically increased cytolytic activity of tumor specific $\mathrm{T}$ lymphocytes after radio-frequency ablation in patients with hepatocellular carcinoma and colorectal liver metastases," 
World Journal of Gastroenterology, vol. 12, no. 23, pp. 3716-3721, 2006.

[94] C. Napoletano, F. Taurino, M. Biffoni et al., "RFA strongly modulates the immune system and anti-tumor immune responses in metastatic liver patients," International Journal of Oncology, vol. 32, no. 2, pp. 481-490, 2008.

[95] M. Widenmeyer, Y. Shebzukhov, S. P. Haen et al., "Analysis of tumor antigen-specific $\mathrm{T}$ cells and antibodies in cancer patients treated with radiofrequency ablation," International Journal of Cancer, vol. 128, no. 11, pp. 2653-2662, 2011.

[96] S. A. Dromi, M. P. Walsh, S. Herby et al., "Radiofrequency ablation induces antigen-presenting cell infiltration and amplification of weak tumor-induced immunity," Radiology, vol. 251, no. 1, pp. 58-66, 2009.

[97] P. Rovere-Querini and A. A. Manfredi, "Tumor destruction and in situ delivery of antigen presenting cells promote anti-neoplastic immune responses: implications for the immunotherapy of pancreatic cancer," Journal of the Pancreas, vol. 5, no. 4, pp. 308-314, 2004.

[98] S. R. Gameiro, J. P. Higgins, M. R. Dreher et al., "Combination therapy with local radiofrequency ablation and systemic vaccine enhances antitumor immunity and mediates local and distal tumor regression," PLoS ONE, vol. 8, no. 7, Article ID e70417, 2013.

[99] B. Al-Sakere, C. Bernat, F. André et al., "A study of the immunological response to tumor ablation with irreversible electroporation," Technology in Cancer Research \& Treatment, vol. 6, no. 4, pp. 301-305, 2007.

[100] X. Li, K. Xu, W. Li et al., "Immunologic response to tumor ablation with irreversible electroporation," PLoS ONE, vol. 7, no. 11, Article ID e48749, 2012.

[101] R. E. Neal II, J. H. Rossmeisl Jr., J. L. Robertson et al., "Improved local and systemic anti-tumor efficacy for irreversible electroporation in immunocompetent versus immunodeficient mice," PLoS ONE, vol. 8, no. 5, Article ID e64559, 2013.

[102] P. McCulloch, D. G. Altman, W. B. Campbell et al., "No surgical innovation without evaluation: the IDEAL recommendations," The Lancet, vol. 374, no. 9695, pp. 1105-1112, 2009. 


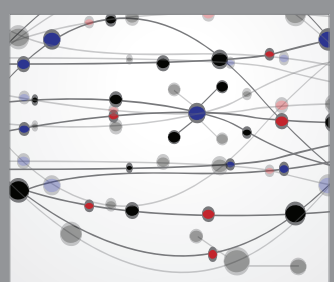

The Scientific World Journal
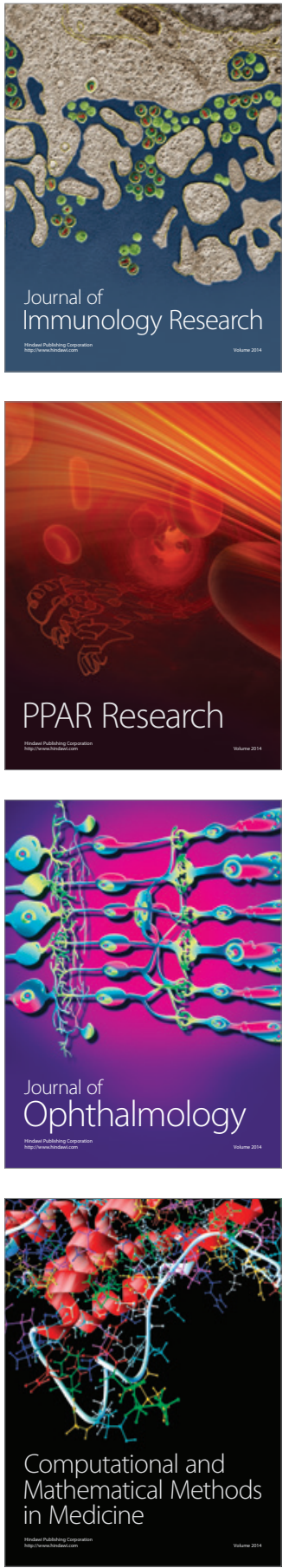

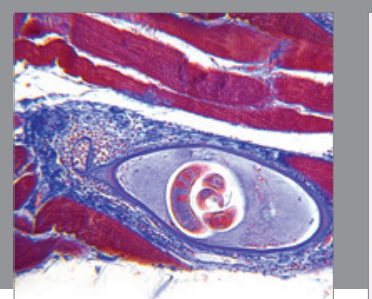

Gastroenterology Research and Practice

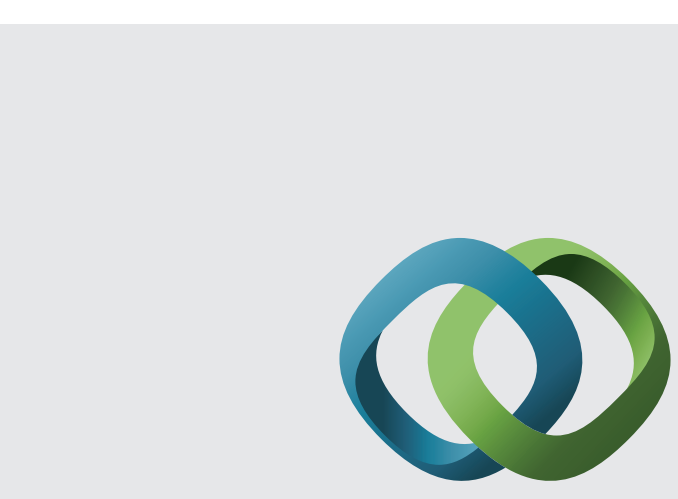

\section{Hindawi}

Submit your manuscripts at

http://www.hindawi.com
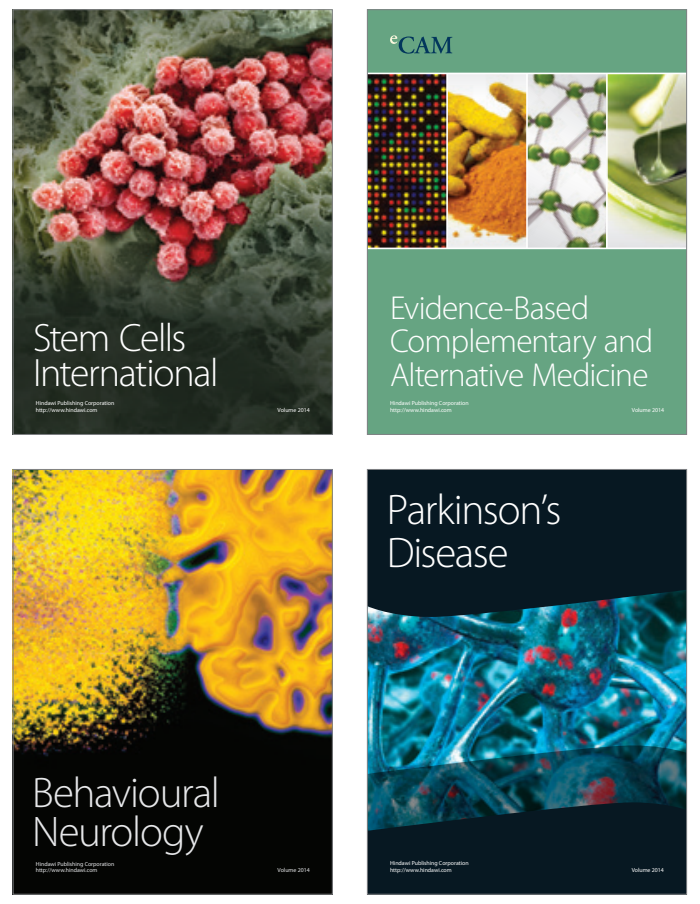
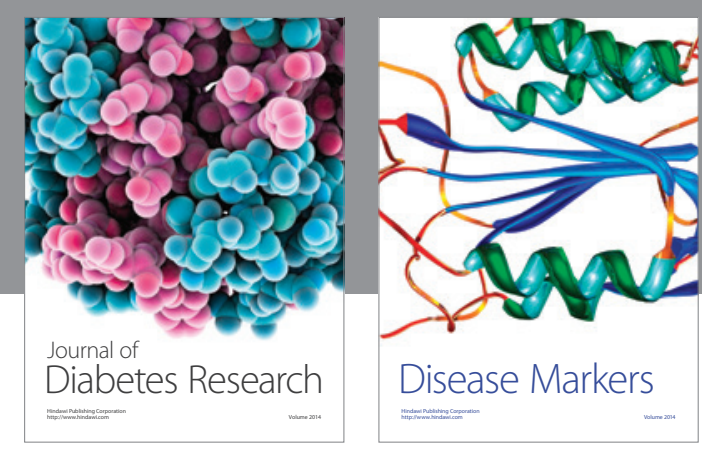

Disease Markers
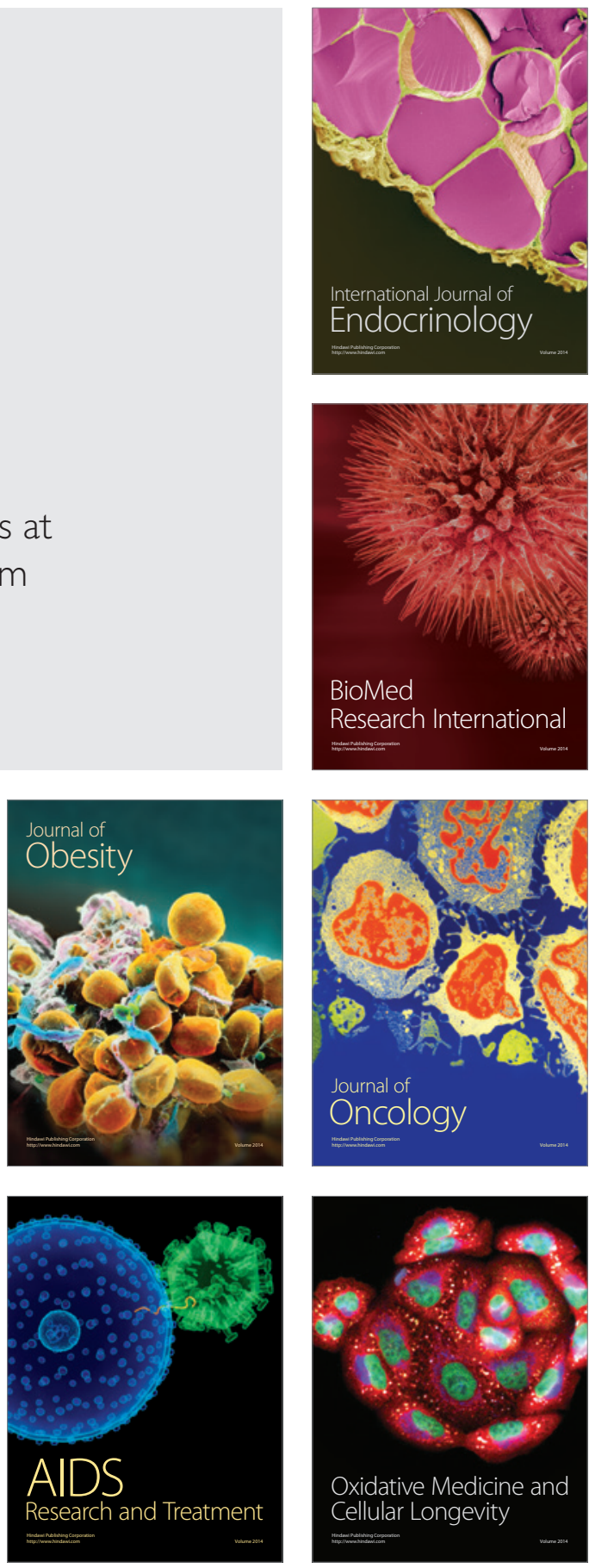